\title{
EPIDEMIOLOGIA
}

\section{Prevención de la infección intrahospitalaria por Staphylococcus aureus resistente a meticilina mediante el manejo de portadores}

\author{
CARMEN MENDOZA N. ${ }^{1}$, CRISTIAN BARRIENTOS M. ${ }^{2}$, E.U. VALENTINA PANIZZA F.${ }^{3}$, \\ E.U. BEATRIZ CONCHA R. ${ }^{4}$, PATRICIO ROMERO P. ${ }^{4}$, T.M. M. CRISTINA BARAHONA F. ${ }^{1}$, \\ T.M. ERNA RAHMANN P. ${ }^{1}$ y T.M. SERGIO MONTEALEGRE M. ${ }^{1}$
}

\section{PREVENTION OF METHICILLIN RESISTANT Staphylococcus aureus NOSOCOMIAL INFECTION BY ELIMINATION OF CARRIERS}

Methicillin resistant Staphylococcus aureus (MRSA) nosocomial infection is very prevalent and has important therapeutic and economic implications. $\boldsymbol{S}$ aureus carriers appear to play the major role on its pathogenesis, they can be part of the medical team as well as patients. In this trial we studied $\mathbf{S}$. aureus and MRSA carriers on the pediatric ICU's medical team and patients. The medical team had $28.8 \%$ (n: 13) of S. aureus carriage and $8.8 \%$ (n: 4) of MRSA on a nasal culture. On the other hand, patients were $33.5 \%$ (n: 43) positive at different hospitalization periods for $\mathbf{S}$. aureus and 16,4\% (n: 21) for MRSA. All of MRSA carriers were treated with nasal mupirocin. After the first treatment cycle we found $100 \%$ eradication on the medical team group, and $85.7 \%$ on patients, which increased to $100 \%$ after the second treatment cycle. The ICU's MRSA nosocomial infection rate was significantly reduced during this study, from $5.19 \%$ (1998) to $0.57 \%$ (1999 first semester). We concluded that carrier treatment confers an important benefit, but it must be always associated to general measures for infection control, such a good hand scrubing.

Key words: Methicillin resistant Staphylococcus aureus (MRSA); Carrier treatment; MRSA nosocomial infection prevention; Nasal mupirocin.

\section{INTRODUCCION}

Staphylococcus aureus es el agente más frecuentemente identificado como causa de infección nosocomial, particularmente $S$. aureus re- sistente a meticilina (SAMR). En nuestro hospital, durante 1998, SAMR representó el 69\% de las cepas de Staphylococcus aisladas en cuadros de infección intrahospitalaria (IIH) y tuvo una incidencia de 5,19 por 100 egresos en la

\footnotetext{
Hospital Doctor Exequiel González Cortés:

${ }^{1}$ Laboratorio de Microbiología.

${ }^{2}$ Estudiante de Medicina. Universidad de Chile.

${ }^{3}$ Control de IIH.

${ }^{4}$ Unidad de Cuidados Intensivos.
} 
Unidad de Cuidados Intensivos (UCI), lo que representa una importante proporción de las infecciones ocurridas en esa unidad, con una tasa global de 21,03 por 100 egresos. Las localizaciones fueron diversas y habitualmente determinaron enfermedad grave, como neumonía asociada a ventilación mecánica, peritonitis, bacteremia asociada a catéter venoso central y nutrición parenteral, infección de herida operatoria, etc. ${ }^{1}$

La infección por SAMR representa, además de su repercusión en la morbimortalidad de los pacientes, un impacto económico importante para el hospital. El espectro de antimicrobianos útiles para su manejo está prácticamente reducido a vancomicina, antibiótico de alto costo y cuya efectividad depende de la eventual adquisición de factores de resistencia desde otras bacterias ya resistentes y que pueden transferírselos. ${ }^{2,3}$

Es conocido el rol que juegan las manos del personal en la transmisión de SAMR en el hospital, al transferirlo entre pacientes o desde la propia flora de piel o mucosas, considerando que la portación en este grupo de individuos es, según diversas publicaciones, mayor que en la población general. , $^{2,4,5}$

Hasta hace pocos años no se disponía de un tratamiento efectivo en la erradicación de portadores; los que se probaron no evitaron la rápida recolonización de los individuos. Sin embargo, actualmente se dispone de mupirocina tópica que ha demostrado seguridad y eficacia en el manejo de este problema. ${ }^{6,7}$

El propósito de esta investigación es determinar la frecuencia de portadores de SAMR en pacientes y personal de la UCI del Hospital Dr. E. González Cortés y su importancia en el desarrollo de la IIH por este agente y probar la eficacia de un antimicrobiano tópico, mupirocina endonasal, en la erradicación del microorganismo y prolongación de este beneficio en el largo plazo.

\section{MATERIAL Y METODO}

El estudio se desarrolló en la Unidad de Cuidados Intensivos del Hospital Dr. E. González Cortés. Se identificó a los funcionarios portadores de SAMR mediante hisopado nasal, que se realizó con tórula de algodón frotada sobre la mucosa de ambas aletas nasales. Las muestras obtenidas fueron transportadas de inmediato al laboratorio, donde se sembraron en agar sangre y posteriormente se identificó el microorganismo de acuerdo a recomendaciones internacionales, sometiendo las cepas de $S$. aureus a estudio de sensibilidad mediante técnica de Kirby-Bauer con disco de oxacilina de $1 \mu \mathrm{g}$.

Los miembros del personal portadores de SAMR fueron tratados con mupirocina endonasal (BACTROBAN nasal®) 2 veces al día durante 5 días según instrucciones entregadas por el fabricante, y controlados mediante cultivo a las 48 horas de terminado el tratamiento y seis meses después.

Simultáneamente se estudiaron todos los pacientes que se hospitalizaron en la unidad en un período de seis meses, mediante cultivo nasal realizado con hisopado como el descrito, al ingreso, al $4^{\circ}$ día y posteriormente cada 7 días hasta su egreso, de modo de identificarlos en el momento en que se hacían portadores de esta bacteria, indicándoles tratamiento con mupirocina endonasal en la forma señalada, en el momento en que un cultivo era positivo a SAMR.

Durante el desarrollo de esta investigación se mantuvo una estricta vigilancia de las infecciones intercurrentes que presentan estos pacientes, calculando las tasas de incidencia de infección por SAMR, comparándolas con las tasas históricas de la unidad.

La evaluación de los resultados se realizó mediante la prueba de significación estadística de $t$ student.

El protocolo de este trabajo fue presentado y aprobado por el Comité de Etica del establecimiento.

\section{RESULTADOS}

Personal: Se estudiaron 45 funcionarios del equipo de salud (10 médicos, 9 enfermeras, 6 kinesiólogos, 18 técnicos paramédicos y 2 auxiliares) aislándose $S$. aureus en 13 de ellos $(28,8 \%)$ y SAMR en $4(8,8 \%)$. Estos 4 funcionarios ( 2 enfermeras, 1 kinesiólogo y 1 técnico paramédico) se trataron con mupirocina 
Tabla 1. Distribución de los pacientes estudiados según grupo de edad

\begin{tabular}{lrr}
\hline Grupo etario & n & \% \\
\hline Recién nacido & 4 & 3,1 \\
Lactante menor & 30 & 23,4 \\
Lactante mayor & 18 & 14,1 \\
Preescolar & 36 & 28,1 \\
Escolar & 40 & 31,2 \\
& & \\
Total & 128 & 100,0 \\
\hline
\end{tabular}

endonasal. El control a las 48 horas de finalizado el tratamiento reveló ausencia de $S$. aureus en todos ellos, es decir, la erradicación fue 100\%. El control a los 6 meses no mostró recolonización en ninguno de ellos.

Pacientes: Se incluyeron 128 pacientes, cuya distribución etaria se presenta en la Tabla 1.

En los 128 pacientes se realizaron 246 hisopados nasales, con un máximo de 9 muestras en 2 casos.

Se aisló $S$. aureus en 43 pacientes $(33,5 \%)$ y SAMR en $21(16,4 \%)$. Ocho de estos pacientes portaban SAMR en el momento de la primera muestra. Tres de ellos había ingresado directamente a UCI y 5 correspondían a traslados desde otras unidades, con períodos previos de hospitalización entre 2 y 24 días.

De los restantes 13 pacientes, 9 adquirieron SAMR al cuarto día y 4 entre el $11^{\circ}$ y $46^{\circ}$ día de hospitalización en UCI.

El tratamiento con mupirocina endonasal se pudo evaluar en 14 de los 21 pacientes. Se logró la erradicación de SAMR en 12 (85,7\%), permaneciendo positivos a SAMR 2 pacientes, que negativizaron el cultivo con un segundo ciclo de administración del fármaco.

La tasa de IIH por SAMR en UCI en el período del estudio fue de $0,57 \%$ ( 1 caso en 173 egresos), significativamente inferior a la calculada para el año anterior (Tabla 2).

\section{DISCUSION}

$S$. aureus es un agente ampliamente diseminado constituyendo parte de la flora comensal del ser humano, principalmente en la piel de axilas, ingles, periné y fosas nasales., ${ }^{2,7}$

Los niños se colonizan rápidamente después del nacimiento, con 6 a 24\% de portación a los 3 ó 4 meses de vida. En los adultos sanos se describen tasas de portación de 11 a $32 \%$, pudiendo ser mayor en el personal hospitalario. ${ }^{2}$ 4,7

La portación nasal en el personal hospitalario y pacientes varía de acuerdo a diversos factores propios de la población en estudio y puede ser permanente o intermitente, y tanto de cepas susceptibles como resistentes a los antimicrobianos. ${ }^{3,7}$

La portación nasal ha sido reconocida como importante en la epidemiología de la infección estafilocóccica desde hace décadas, siendo el mejor indicador de colonización y potencial diseminación del agente. Constituye el principal reservorio del microorganismo, desde donde coloniza las otras áreas de la piel y las manos del personal de salud, ${ }^{2,3,8,13}$ que son el vehículo de contagio a otros pacientes.

En nuestro estudio encontramos una tasa de portación nasal de $S$. aureus en funcionarios de salud que se aproxima al $30 \%$ descrito en

Tabla 2. Tasas de infección intrahospitalaria por Staphylococcus aureus resistentes a meticilina en Unidad de Cuidados Intensivos.

Hospital. E. González Cortés. Año 1998 y primer semestre 1999

\begin{tabular}{lccc}
\hline Año & $\mathbf{N}^{\circ}$ casos & Egresos & Tasa (\%) \\
\hline 1998 & 20 & 385 & 5,19 \\
$1999\left(1^{\circ}\right.$ semestre $)$ & 1 & 173 & 0,57 \\
$\mathrm{P}<0,001$ (t student). & & & \\
\hline
\end{tabular}


otros estudios, correspondiendo un tercio de las cepas a SAMR.

Por otra parte, el paciente portador nasal de $S$. aureus puede desarrollar cuadros infecciosos de diversas localizaciones a partir de esta colonización, como se ha visto en pacientes operados en que hay autoinoculación de herida, en hemodializados que desarrollan bacteremia, etc e $, 7,14^{2}$

Algunos estudios demuestran que $90 \%$ de los infectados por $S$. aureus en UCI son portadores de la misma cepa, y si el agente portado corresponde a una cepa resistente a meticilina, el riesgo sería aún mayor. ${ }^{6,9,10}$

En nuestro estudio se encontró en los pacientes $33,5 \%$ de portación nasal en diferentes períodos de su permanencia en la unidad, correspondiendo 48,8\% (21 casos) a SAMR.

La portación nasal de SAMR no es una situación restringida al ambiente hospitalario, ya que en la comunidad existe este agente, ${ }^{12}$ como queda también demostrado en nuestra serie, en que $38 \%$ de los casos lo portaba al momento del ingreso. Esto debe hacer pensar que no sólo implican riesgo los pacientes hospitalizados por largos períodos.

$S$. aureus es el agente más frecuentemente aislado en la patología infecciosa nosocomial $\mathrm{y}$ especial importancia ha adquirido en esta patología SAMR, que en nuestro medio presenta resistencia múltiple, haciendo especialmente difícil su manejo. Prácticamente la única alternativa terapéutica es vancomicina, antimicrobiano de alto costo, no exento de toxicidad, a lo que se agrega además la prolongación de la hospitalización, instauración de medidas de aislamiento, reforzamiento de los programas de vigilancia, etc., que encarecen aún más la atención. ${ }^{6}{ }^{10}$ Esto pone de relevancia la necesidad de actuar con un criterio preventivo, en que el manejo y tratamiento de portadores tiene un lugar importante.

En nuestra experiencia, durante los seis meses que duró el estudio, la IIH por SAMR mostró un descenso altamente significativo con relación a la tasa de IIH durante 1998 y la única infección intercurrente por SAMR fue una neumonía asociada a ventilación mecánica en una paciente portadora de SAMR al ingre- so, que no alcanzó a tratarse antes del inicio de la infección respiratoria.

En la investigación de medidas eficaces para el tratamiento de portadores, desde hace varias décadas se han ensayado diferentes esquemas, ya sea tópicos, con nebulizaciones u orales, los que no mostraron utilidad en la erradicación de $S$. aureus, o aquellos que parecían ser útiles eran tóxicos o inducían el desarrollo de resistencia al agente usado. ${ }^{6,7,13}$

Mupirocina fue introducida a mediados de los años ochenta en el Reino Unido, demostrando ser altamente efectiva en la erradicación de los portadores nasales de $S$. aureus, tanto susceptible como resistente a meticilina, siendo además bien tolerado.

En E.U.A. fue licenciada una formulación de mupirocina cálcica específicamente para uso endonasal (Bactroban nasal ${ }^{\circledR}$, de SmithKline Beecham Pharmaceuticals, Philadelphia, E.U.A.) lo que ha renovado el interés en la erradicación de portadores.

En nuestra experiencia el tratamiento con mupirocina endonasal fue efectivo en $100 \%$ de los funcionarios colonizados con SAMR, con erradicación mantenida al menos hasta 6 meses, y en $87,5 \%$ de los pacientes colonizados con SAMR con la primera aplicación, permaneciendo positivos dos casos que se negativizaron en un segundo tratamiento.

Se ha demostrado que el tratamiento de portadores disminuye el riesgo de desarrollar infecciones como bacteremia en hemodializados, peritonitis e infección del sitio de inserción del catéter en peritoneo-diálisis e infecciones invasoras por $S$. aureus en pacientes con SIDA o portadores de VIH. $6,7,13$

La erradicación de portadores nasales también puede ser un medio para reducir la diseminación del microorganismo de un individuo a otro. ${ }^{6}$

En hospitales de alta endemia o epidemias de infección por SAMR, el tratamiento de personal y pacientes colonizados se considera adecuado, asociado a otras medidas de control, ${ }^{6}$ sin embargo, en hospitales con baja prevalencia en que se ha usado el tratamiento de rutina de pacientes y personal portadores de SAMR, se ha observado desarrollo de resistencia. ${ }^{6,7}$ 
No se ha demostrado resistencia si se usan tratamientos cortos de 5 días, aunque ésta aparece cuando se usa en aplicaciones continuas por períodos de semanas o meses, especialmente en áreas extensas de dermatitis o heridas.

El tratamiento de portadores no es una estrategia válida por sí sola, y debe asociarse con adecuado lavado de manos y medidas de higiene que establezcan barreras más eficientes. ${ }^{7}$ En nuestra observación se realizó, además del tratamiento de portadores, educación en servicio a todos los funcionarios de la unidad con reforzamiento de una adecuada técnica de lavado de manos.

\section{CONCLUSIONES}

Se demostró portación nasal de SAMR en $8,8 \%$ de los funcionarios de la UCI y en $16,4 \%$ de los pacientes.

La portación nasal de SAMR no parece ser un fenómeno limitado al ambiente hospitalario, ya que una proporción importante de los pacientes es portadora al ingreso.

Con el uso de mupirocina endonasal se erradicó al $100 \%$ de los portadores funcionarios y $85,7 \%$ de los portadores pacientes, obteniéndose $100 \%$ de erradicación en este último grupo con una segunda aplicación del antimicrobiano. La erradicación se mantuvo durante todo el período estudiado.

Las limitaciones de esta experiencia radican en el bajo número de pacientes con control del tratamiento de erradicación, y para resultados más definitivos se requerirá de un mayor número de casos incluidos en la muestra.

El tratamiento de portadores, junto a una política de lavado de manos adecuado puede reducir drásticamente la incidencia de infección intrahospitalaria por este agente, por lo que parece recomendable la vigilancia permanente (semestral) del personal de salud y si la situación epidemiológica lo justifica, realizar el tratamiento de los portadores con mupirocina endonasal, que ha demostrado ser altamente efectiva en la erradicación de SAMR. Los pacientes deben vigilarse desde el ingreso y luego semanalmente, tratándose aquellos casos posi- tivos con el esquema de mupirocina endonasal usado en esta experiencia.

\section{RESUMEN}

La infección intrahospitalaria (IIH) por SAMR tiene una alta prevalencia e implicancias terapéuticas y económicas. En la patogenia de la infección tienen importancia los portadores del agente, tanto funcionarios de salud como pacientes. En este estudio se realizó una investigación de portadores nasales de Staphylococcus aureus y de SAMR en funcionarios de la unidad de cuidados intensivos, que mostró una tasa de $28,8 \%$ (n: 13 ) y $8,8 \%$ (n: 4) respectivamente. Los pacientes presentaron una tasa de portación de $S$. aureus de 33,5\% (n: 43) y de SAMR de $16,4 \%$ (n: 21), en diferentes períodos de hospitalización.

Todos los portadores de SAMR se trataron con mupirocina endonasal, obteniéndose $100 \%$ de erradicación con un ciclo de tratamiento en funcionarios y $85,7 \%$ en pacientes, en los cuales se logró $100 \%$ de erradicación con un segundo ciclo de tratamiento.

La tasa de IIH por SAMR en la UCI durante el período de estudio descendió significativamente desde $5,19 \%$ (año 1998) a $0,57 \%$ (primer semestre 1999).

Se concluye que el tratamiento de portadores reporta un importante beneficio, debiendo asociarse a otras medidas de control, como un adecuado lavado de manos en la atención de pacientes.

\section{BIBLIOGRAFIA}

1.- Registros de la Vigilancia de Infecciones Intrahospitalarias. Comité de Infecciones Intrahospitalarias, Hospital Dr. E. González Cortés.

2.- MARANAN M, MOREIRA B, BOYLE-VAVRA $S$, DAUM R. Antimicrobial resistance in Staphylococci. Epidemiology, Molecular Mechanism and Clinical Relevance. Infect Dis Clin North Amer 1997; 11: 813-49.

3.- HUDSON I. The efficacy of intranasal mupirocin in the prevention of staphylococcal infection: a review of recent experience. J Hosp Infect 1994; 27: 81-9.

4.- MADRID P, HERING E, KÄGGI M et al. Inciden- 
cia de la portación nasal de Staphylococcus aureus y de su resistencia a oxacilina en pacientes con uno y tres días de hospitalización. Calvo Mackenna. Medicina y Cirugía del Niño 1996; 2: 53-6.

5.- DOEBBELING B, BRENEMAN D, NEU H et al. Elimination of Staphylococcus aureus nasal carriage in health care workers: Analysis of six clinical trials with calcium mupirocin ointment. CID 1993; 17: 466-74.

6.- BOYCE J. Preventing staphylococcal infections by eradicating nasal carriage of Staphylococcus aureus: Proceeding with caution. Infect Control Hosp Epidemiol 1996; 17: 775-9.

7.- SCHMITZ F, JONES M. Antibiotics for treatment of infection caused by MRSA and elimination of MRSA carriage. What are the choices? Int J Antimicrob Agents 1997; 9: 1-19.

8.- LEDERMANN W. Portación nasal de Staphylococcus aureus. Calvomackenna. Medicina y Cirugía del Niño 1996; 2: 41-5.

9.- WALDVOGEL F. Staphylococcus aureus. (Including Staphylococcal Toxic Shock). Mandell, Douglas and Bennett's Principles and Practice of Infectious Diseases. Mandell GL, Bennett J E, Dolin
R. eds. Fifth ed 2000, Churchill Livingstone, Philadelphia, Chapter 183, pp 2069-91.

10.- HERWALDT L. Conferencia: Infecciones por Staphylococcus aureus meticilina resistente, un modelo vivo. Curso: La infectología en el control de las infecciones intrahospitalarias. Sociedad Chilena de Infectología. Septiembre de 1998. Santiago de Chile.

11.- LEDERMANN W. Lenguaje infectológico. Rev Chil Infect 1999; 16: 241-2.

12.- CHAVEZ A, BAHAMONDES D, PAYA E, MENDOZA C, PAPIC Z. Infecciones por Staphylococcus aureus meticilina resistente. Experiencia clínica. Rev Chil Infect 1995; 12: 152-6.

13.- LEDERMANN W. Portación de Staphylococcus aureus. Rev Chil Infect 1996; 13: 81-4.

Agradecimientos: Al voluntariado Damas de Calipso del Hospital Dr. Exequiel González Cortés por el financiamiento de los procedimientos de laboratorio de microbiología aplicados en este estudio. A Laboratorio SmithKline Beecham, que aportó las dosis de Bactroban nasal® requeridas para el tratamiento de los portadores.

Correspondencia a:

Carmen Mendoza N.

Simón Bolivar 7858 I, La Reina, Santiago, Chile.

Fono: (56-2) 2734015

Fax: (56-2) 2751842

Email: bym@rdc.cl 